\title{
Flu virus finally sequenced in its native form
}

\section{Technique could help to unpick role of enigmatic chemical modifications in genetic material.}

\section{BY EWEN CALLAWAY}

$\mathrm{T}$ The genome of the flu virus has been fully sequenced in its native RNA form for the first time. Previously, all influenza genomes - as well as those of other viruses that store their genetic material as RNA — had been determined by copying the molecule into DNA. The native flu genome was generated using 'nanopore' sequencing technology, which reads RNA strands as they stream through a tiny molecular channel.

"For the first time, we can really start to look at the nature of the genome in its original state," says John Barnes, a microbiologist at the US Centers for Disease Control and Prevention (CDC) in Atlanta, Georgia, who led the effort, described in a preprint posted ${ }^{1}$ to the bioRxiv server on 12 April. "It really does start to open up a lot of possibilities."

Barnes and other scientists are most interested in probing viral genomes, as well as other forms of RNA in many types of organism, including humans. Researchers want to investigate mysterious chemical ornaments on RNA molecules that might affect their function in cells but have been hard to study. "The real excitement here is about RNA modifications," says Ewan Birney, co-director of the European Bioinformatics Institute in Hinxton, UK. The approach is "transformative", says Birney.

RNA is chemically similar to its betterknown cousin, DNA. In cellular organisms, it serves as an intermediary between DNAencoded genes and proteins, and performs other tasks. But many viruses - including those behind polio, Ebola and the common cold - store their genetic information as RNA. Barnes, head of the CDC's influenza-genomics team, says that no one had sequenced the virus's RNA genome before because it seemed nearly impossible. Previous methods for sequencing native RNA strands involved degrading one chemical base, or letter, at a time, and these techniques have changed little since their invention in the late $1970 \mathrm{~s}^{2}$. To compensate, nearly all 'RNA sequencing' instead uses a viral enzyme called reverse transcriptase, which copies RNA into sequencer-friendly DNA strands.

Nanopores offer a simpler way of sequencing actual RNA molecules, such as viral genomes. The technology is based on applying electrical

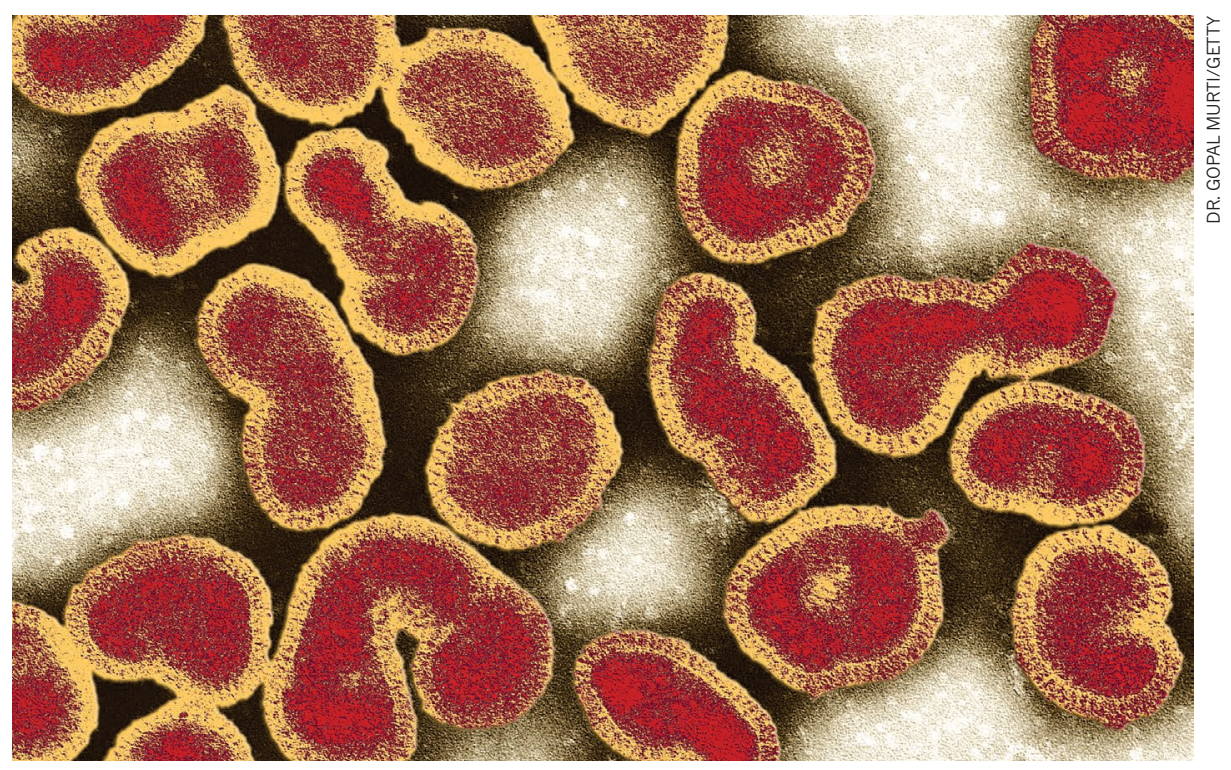

The influenza virus is the first RNA virus to have been sequenced in its original state.

current across a nanoscale molecular pore, and then measuring telltale current fluctuations as genetic material snakes through.

\section{TINY IS MIGHTY}

In January, researchers at a leading developer of the technology, UK-based Oxford Nanopore Technologies, directly sequenced RNA using a small device called the MinION ${ }^{3}$. That effort looked at transcripts of messenger RNA, the family of "It really does RNA molecules that conveys information start to open up a lot of possibilities." from DNA to build proteins.

Barnes's team applied this method to the genome of influenza $\mathrm{A}$, which is roughly 13,500 RNA letters long and composed of 8 segments. Barnes says his team's approach isn't ready for public use. It required a lot of flu virus and, to iron out sequencing errors, the raw data had to be processed many times. But nanopore technology is advancing quickly, and Barnes hopes that with improvements, direct sequencing of RNA viruses will become routine.

At the top of his and other scientists' wish lists are methods for identifying chemical modifications to RNA. More than 100 have been identified so far, but researchers have little idea what most of them do, in large part because it has been impossible to study them systematically. The Oxford Nanopore team was able to detect two common RNA modifications, or tags. Birney, who is a paid consultant to the company, expects that the technology will be able to find many more once machinelearning algorithms are used to unpick the tags' signatures.

Sequencing modified bases of RNA would be "a big deal" for the field, says Bryan Cullen, a virologist at Duke University in Durham, North Carolina. His team found last year ${ }^{4}$ that during infection in mice, a tag called $\mathrm{m}^{6} \mathrm{~A}$ seems to alter the expression of influenza genes to promote viral replication. Current methods for detecting such modifications are time-consuming and expensive, he adds.

Although the methods aren't yet perfect, Birney says, biologists are still excited about the possibility of soon being able to sequence entire viral genomes and other RNA molecules in their natural forms. "Suddenly, we've got the technology to do this. It's kind of amazing." -

1. Keller, M. W. et al. Preprint at bioRxiv https://doi. org/10.1101/300384 (2018).

2. Peattie, D. A. Proc. Natl Acad. Sci. USA 76, 1760 1764 (1979).

3. Garalde, D. R. et al. Nature Meth. 15, 201-206 (2018).

4. Courtney, D. G. et al. Cell Host Microbe 22, 377-386. e5 (2017). 